\title{
Dolor crónico nociceptivo y neuropático en población adulta de Manizales (Colombia)
}

\section{Chronic nociceptive and neuropathic pain in adult population in Manizales (Colombia)}

\author{
Ricardo Díaz, Felipe Marulanda • Manizales
}

\section{Resumen}

Introducción: el dolor crónico es uno de los síntomas que más afectan la funcionalidad y calidad de vida de quienes lo padecen. En Colombia son escasos los estudios sobre su comportamiento epidemiológico y clínico.

Objetivo: estimar la prevalencia y aspectos clínicos del dolor crónico nociceptivo y neuropático en una muestra representativa de la ciudad de Manizales.

Metodología: se entrevistaron 627 personas mayores de 18 años de ambos sexos, seleccionadas al azar en diferentes barrios de la ciudad de Manizales, aplicando un cuestionario semiestructurado, mediante entrevista domiciliaria realizada por estudiantes de la Facultad de Ciencias para la Salud. La información fue procesada mediante el paquete estadístico Epi-Info.

Resultados: se encontró una prevalencia de dolor crónico de $33.9 \%$, correspondiendo al tipo nociceptivo el $31.4 \%$ y al neuropático el $2.5 \%$. Hubo predominio del género femenino para ambos tipos de dolor y para el nociceptivo la frecuencia aumentó con la edad. La localización más frecuente fue la cabeza en el nociceptivo y los miembros superiores para el neuropático. La duración del dolor fue mayor a un año en dos terceras partes de los sujetos y superior a cinco años en la tercera parte de ellos. La mitad de las personas con dolor crónico reportaron compromiso significativo del estado de ánimo, y una tercera parte afectación del patrón de sueño. La medicación predominante en ambos tipos de dolor fueron AINES y acetaminofén, con escaso consumo de opiodes. El $41 \%$ de los entrevistados reportó autoformulación. La escala de tamizaje de dolor neuropático (DN4) resultó útil para la identificación de sujetos con este tipo de dolor y mostró capacidad de discriminación frente al dolor nociceptivo, dependiendo de la inclusión o no de los items del examen físico.

Conclusiones: esta investigación confirma la alta prevalencia de dolor crónico en la población adulta y su tendencia a persistir a través de los años, con repercusión negativa en los aspectos emocionales, sueño y calidad de vida. Se resalta la necesidad de un adecuado diagnóstico y manejo individual, apropiadas políticas en salud y permanente desarrollo investigativo para enfrentar esta importante condición (Acta Med Colomb 2011; 36: 10-17).

Palabras clave: epidemiología, dolor crónico, nociceptivo, neuropático, autoformulación, adultos.

\begin{abstract}
Introduction: chronic pain is one of the symptoms that most affect the functionality and quality of life of its sufferers. In Colombia, there are few studies on its epidemiological and clinical behavior.

Objective: to estimate the prevalence and clinical aspects of chronic nociceptive and neuropathic pain in a representative sample of the city of Manizales.

Methods: we interviewed 627 people of both sexes, aged 18 years and over, randomly selected in several districts of the city of Manizales, using a semi-structured home interview conducted by students of the Faculty of Health Sciences. The information was processed using the statistical package Epi-Info.

Results: the prevalence of chronic pain was found to be $33.9 \%, 31.4 \%$ nociceptive and $2.5 \%$ neuropathic. There was predominance in the female sex for both types, and for nociceptive pain the frequency increased with age. The most common location were the head for nociceptive pain and the upper limbs for neuropathic pain. The duration of pain was more than one year in two thirds of the subjects, and over 5 years in a third of them. Half of the people with chronic pain reported significant
\end{abstract}

Dr. Ricardo Díaz Cabezas: Neurólogo Clínico. Profesor Asociado U. de Caldas; Dr. Felipe Marulanda Mejía: Geriatra Clínico. Profesor Asociado U. de Caldas. Manizales. Correspondencia: Ricardo Díaz Cabezas. Clínica de la Presentación Cra 23 Cll 46 Centro Médico. Manizales, Caldas

E-mail: ridiaz@une.net.co

Recibido: 18/I/2011 Aceptado: 14/II/2011 
mood alteration, and one third reported involvement in their sleep pattern. The predominant drugs in both types of pain were NSAIDs and acetaminophen, with low consumption of opioids. $41 \%$ of respondents reported self-medication. The scale screening of neuropathic pain (DN4) was useful for identifying individuals with this type of pain, and showed the ability to discriminate nocicceptive pain, depending on the inclusion or exclusion of items of physical examination.

Conclusions: this study confirms the high prevalence of chronic pain in the adult population and its tendency to persist over the years, with involvement in emotional aspects and quality of life. It also shows the clinical utility of screening tests for differentiation of types of chronic pain. This highlights the need for proper diagnosis and management of individual patients, as well as for an appropriate health policy and the development of research to meet this condition (Acta Med Colomb 2011; 36: 10-17).

Keywords: epidemiology, chronic pain, nociceptive, neuropathic, self-medication, adults.

\section{Introducción}

El dolor crónico constituye un problema de salud que afecta seriamente al individuo, repercutiendo sobre la funcionalidad, el bienestar emocional y la calidad de vida, con consecuencias negativas sobre la familia y el entorno social. Además, tiene impacto en la seguridad social comprometiendo recursos y generando mayores costos.

La IASP ha definido dolor crónico como aquel que dura tres meses o más y dolor agudo aquel que tiene duración menor a tres meses (1). Según el mecanismo fisiopatológico se ha clasificado en dolor nociceptivo, que como su nombre lo dice se genera a partir de estímulos de nociceptores; éste a su vez se puede subdividir en óseo, muscular, visceral y superficial o dérmico y en dolor neuropático, que a su vez puede ser de origen central y periférico.

El dolor es la causa más frecuente de consulta. Algunas estadísticas señalan que cerca del 35 al 50\% de la población general padece de dolor crónico. Un estudio reciente de Torrance et al (2) detectó que la prevalencia de dolor crónico de cualquier origen en la población general es del 48\%, mientras que el de origen neuropático es del $8 \%$. Este último tipo de dolor puede aumentar a un $27 \%$ en pacientes atendidos en clínicas de dolor y a $36 \%$ en aquéllos con cáncer.

Un estudio europeo multicéntrico que incluyó 16 países (3) mostró que una quinta parte de adultos europeos, padecen dolor crónico moderado a severo y en muchos de éstos los síntomas son manejados de manera inadecuada. El $19 \%$ de los encuestados padecía dolor crónico de moderado a severo que afectaba seriamente sus actividades laborales, sociales y de vida diaria. Un 59\% había tenido dolor entre 2 y 15 años, al $21 \%$ se le había dado un diagnóstico de depresión debido a su dolor, $61 \%$ eran incapaces de trabajar fuera de casa, $19 \%$ habían perdido sus empleos, y $13 \%$ lo habían cambiado a causa del dolor. En cuanto a medicamentos prescritos, los más consumidos fueron los AINES en $44 \%$, opioides débiles $23 \%$, acetaminofén $18 \%$, cox-2 $6 \%$ y opioides fuertes $5 \%$.

Un estudio similar realizado en España (4) mostró como localizaciones más frecuentes, espalda $(50.9 \%)$, cabeza (42\%) y piernas $(36.8 \%)$. El dolor fue muy severo o intolerable en el $33 \%$ de la muestra, siendo mujeres y ancianos quienes presentaron las mayores intensidades. Las activi- dades sociales y personales se vieron afectadas en $25.4 \%$ de los casos y un $10.4 \%$ se incapacitaron completamente.

En Colombia la Asociación Colombiana para el Estudio del Dolor (ACED) publicó en abril de 2004 la III Encuesta Nacional de Dolor, la cual arrojó las siguientes cifras: el $47.7 \%$ de la población aceptó haber tenido algún tipo de dolor en el último mes, siendo más frecuente la cefalea, seguida del dolor lumbar. De éstos, el 50\% refirieron una duración mayor a un mes y el $52.4 \%$ de los encuestados aceptó que su actividad habitual se había visto interrumpida a causa del mismo. El $64.5 \%$ había consultado a un médico, mientras que el $48 \%$ se había automedicado (5). En la IV Encuesta Nacional de Dolor de la ACED del año 2008 con 1204 individuos entrevistados de manera telefónica, la prevalencia del dolor en el último mes fue del 53\%, predominante en las mujeres, sin hacer alusión a la prevalencia del dolor crónico (6).

Un trabajo reciente llevado a cabo por nuestro grupo de dolor de la Universidad de Caldas acerca del dolor crónico en el Departamento de Caldas (DOLCA), arrojó una prevalencia de dolor en el último mes del $50 \%$ y de dolor crónico del $31 \%$ (7).

La IASP introdujo en el año 1994 la siguiente definición para dolor neuropático (DNe): "Dolor iniciado o causado por una lesión primaria o disfunción en el sistema nervioso" (8); pero en el año 2008 el Grupo de Interés Especial en Dolor Neuropático de la IASP (NeuPSIG) liderado por Treede RD (9) propuso una redefinición del DNe como aquel que "surge como consecuencia directa de una lesión o enfermedad que afecta el sistema somatosensorial". Estos mismos autores proponen un sistema de graduación de la precisión diagnóstica del dolor neuropático, tanto para propósitos clínicos como de investigación, en posible, probable y definitivo.

$\mathrm{El} \mathrm{DNe}$ es causado por varios trastornos periféricos y centrales del sistema nervioso y constituye un grave problema de salud debido a sus características de severidad, cronicidad y refractariedad al manejo con analgésicos comunes.

Múltiples condiciones médicas pueden manifestarse con DNe, siendo las más comunes en la consulta médica, lumbalgia con radiculopatía, neuropatía diabética, neuralgia postherpética, trauma raquimedular y dolor neuropático 
asociado a cáncer. Otras causas menos comunes son síndrome doloroso regional complejo, esclerosis múltiple, dolor posterior a daño cerebrovascular, neuropatía por VIH y neuralgia del trigémino $(10,11)$.

La prevalencia del DNe ha sido informada con cifras variables. Bowsher (12) anotó una prevalencia estimada del $1 \%$ en la población general. Gilron I (13) en su revisión sobre DNe menciona datos de prevalencia del 2 al $3 \%$ en la población general. Estudios más recientes en Europa, empleando una metodología a través de encuestas postales, han encontrado prevalencias de DNe de $8 \%$ en el Reino Unido (2) y de $6.9 \%$ en Francia (14).

En América Latina se estima que el DNe afecta al $2 \%$ de la población general $(15,16)$ y que el $15 \%$ de los pacientes que asisten a consulta médica por dolor, sufren de DNe. En Colombia no se han llevado a cabo estudios poblacionales sobre la prevalencia y características sociodemográficas del DNe. Como se mencionó antes, los estudios realizados por la ACED (Asociación Colombiana para el Estudio del Dolor) denominados Encuesta Nacional de Dolor en los años 2004 y $2008(5,6)$, no hacen mención específica del DNe.

Es usualmente reconocido que el DNe es difícil de tratar y uno de los aspectos principales que afectan el pronóstico es la presencia de comorbilidades como trastornos de sueño, depresión y ansiedad. Los pacientes que sufren dolor crónico experimentan dificultades para iniciar y mantener el sueño. La privación de sueño ha sido asociada con una disminución del umbral al dolor, dolores musculares y rigidez en voluntarios sanos. Las interrelaciones de estos factores son complejas: muchos pacientes con dolor crónico son deprimidos y ansiosos; la privación de sueño puede generar ansiedad; y la depresión puede ser tanto causa como resultado de los trastornos de sueño (17).

\section{Material y métodos}

Se realizó un estudio poblacional, descriptivo y transversal en el área urbana del municipio de Manizales, mediante muestreo aleatorio de las viviendas de diferentes estratos socioeconómicos (1-6) y proporcional al número de habitantes de cada uno de ellos. El cálculo de la muestra se basó en la prevalencia reportada del dolor crónico y la población adulta de este municipio, que para el año 2009 se estimaba alrededor de 300.000 personas.

La información fue obtenida a través de una encuesta domiciliaria realizada puerta a puerta en las viviendas seleccionadas, a personas mayores de 18 años dispuestas a colaborar después de dar consentimiento informado. Las encuestas se diligenciaron en su mayoría los fines de semana con buena aceptación por parte de los entrevistados. Los encargados de realizarla fueron estudiantes de la Facultad de Ciencias para la Salud pertenecientes al semillero de investigación, entrenados por los autores del trabajo.

El instrumento de recolección de información, consistía en una encuesta semiestructurada realizada a quienes informaran tener dolor con duración mayor a tres meses, es decir, catalogado como "dolor crónico". Los datos consignados abarcaron aquéllos de carácter sociodemográfico, aspectos clínicos alusivos al dolor crónico como localización, intensidad, cualidad, duración, frecuencia de presentación, repercusión emocional sobre ansiedad o depresión, impacto sobre el sueño y aspectos sobre manejo farmacológico recibido.

A todos los sujetos con dolor crónico se les aplicó la escala de bienestar emocional, denominada OMS-5. Esta escala "Índice de Bienestar de la Organización Mundial de la Salud" (OMS-5) califica la percepción emocional del individuo, mediante la formulación de cinco preguntas. A mayor puntuación, mayor bienestar emocional. El rango de puntaje oscila entre $0-25$ y se considera un punto de corte de 13 para distinguir entre buena y mala percepción de bienestar.

Adicionalmente, se les aplicó la escala de tamizaje para posible dolor neuropático, denominada DN4. El grupo "French Neuropathic Pain Control", conformado por expertos en el tema, elaboró un cuestionario conocido como DN4 (Dolor Neuropático 4 Preguntas) con el objetivo de ayudar a los médicos no expertos en dolor, a diferenciar entre dolor nociceptivo y neuropático. Las dos primeras preguntas constan de siete items que revelan síntomas o sensaciones con las cuales el paciente describe su dolor (quemazón, frío doloroso, descarga eléctrica, hormigueo, sensación de agujas, entumecimiento y picazón), mientras que las dos últimas preguntas incluyen tres items que ameritan un examen físico de la zona afectada (hipoestesia al tacto, hipoestesia al pinchazo, alodinia). Se responde si o no a cada item y el puntaje máximo es de 10. Un puntaje de cuatro o más está a favor de que dicho dolor sea de tipo neuropático. En algunos estudios, cuya metodología no permite el examen físico de los sujetos, se ha aplicado una versión modificada que incluye únicamente los siete primeros items, y para la cual se ha determinado un punto de corte de tres.

Siguiendo las recomendaciones de la IASP de un sistema de graduación para el dolor neuropático, se considera un caso, posible, aquel con una distribución del dolor neuroanatómicamente plausible e historia clínica sugestiva de lesión o enfermedad del sistema somatosensorial. Como caso probable, se considera aquel que tiene signos sensoriales en un territorio correspondiente a una estructura nerviosa lesionada. Se considera caso definitivo, el que cumple con los dos criterios anteriores y además tiene una prueba diagnóstica confirmatoria de lesión o enfermedad que explica el dolor neuropático, lo cual no se realizó en el presente estudio. Los sujetos con dolor crónico identificados a través de la encuesta general y la escala DN4 como casos posibles de dolor neuropático, fueron evaluados posteriormente en consulta médica por el grupo de clínica de dolor de la Universidad de Caldas, con el fin de precisar un diagnóstico sindromático y topográfico, en aras de establecer casos probables de dolor neuropático y sus potenciales factores etiológicos.

Se obtuvo el aval del Comité de Bioética de la Facultad de Ciencias para la Salud, con el compromiso por parte de 
los autores, del manejo anónimo y responsable de los datos obtenidos. La información se procesó mediante los paquetes estadísticos Epi-Info 6.04d y Epi-Data 3.1. Las variables cualitativas se analizaron por medio de proporciones y las cuantitativas por medio de medias y desviación estándar. Las frecuencias de enfermedad se expresaron en porcentaje. Se consideró una $\mathrm{p}<0.05$ como estadísticamente significativa en los análisis de asociación bivariados.

\section{Resultados}

Se encuestaron 627 personas con edades comprendidas entre 18 y 91 años, en la zona urbana del municipio de Manizales, con un predominio del género femenino (67.3\%), siendo la mayoría de ellas amas de casa. Por rangos de edad la mayor frecuencia correspondió al grupo de 35 a 64 años (58.7\%). En cuanto al estado civil la mayor parte estaba representada por casados y solteros $(77.8 \%)$ y por nivel socioeconómico la mayor proporción estuvo en estratos tres y cuatro. Los datos sociodemográficos de la muestra se aprecian en la Tabla 1.

Del total de encuestados se encontraron 213 personas con dolor crónico, lo cual correspondió al 33.9\% de la muestra. En 197 personas (31.4\%) el dolor crónico se tipificó como nociceptivo, en 57 personas (9\%) se consideró como posible

Tabla 1. Distribución porcentual de población según variables sociodemográficas.

\begin{tabular}{|c|c|}
\hline Variable & $\mathrm{n}=627(\%)$ \\
\hline \multicolumn{2}{|l|}{ Género } \\
\hline Femenino & $422(67.3)$ \\
\hline Masculino & $205(32.7)$ \\
\hline \multicolumn{2}{|l|}{ Edad / Rango de edad } \\
\hline$<25$ & $93(14.8)$ \\
\hline $25-34$ & $89(14.2)$ \\
\hline $35-49$ & $178(28.4)$ \\
\hline $50-64$ & $190(30.3)$ \\
\hline $65-74$ & $49(7.8)$ \\
\hline$\geq 75$ & $28(4.5)$ \\
\hline \multicolumn{2}{|l|}{ Estado civil } \\
\hline Casado & $273(43.5)$ \\
\hline Soltero & $215(34.3)$ \\
\hline Unión libre & $48(7.7)$ \\
\hline Divorciado & $46(7.3)$ \\
\hline Viudo & $45(7.2)$ \\
\hline \multicolumn{2}{|l|}{ Ocupación } \\
\hline Ama de casa & $254(40.5)$ \\
\hline Empleado & $152(24.2)$ \\
\hline Independiente & $82(13.1)$ \\
\hline Estudiante & $63(10.0)$ \\
\hline Pensionado & $55(8.8)$ \\
\hline Desempleado & $31(4.9)$ \\
\hline \multicolumn{2}{|l|}{ Nivel educativo } \\
\hline Bachiller & 305 (48.6) \\
\hline Profesional/Tecnólogo & $179(28.5)$ \\
\hline Primaria & 137 (21.9) \\
\hline Analfabeta & $6(1.0)$ \\
\hline \multicolumn{2}{|l|}{ Estrato } \\
\hline Estratos 1-2 & $82(13.1)$ \\
\hline Estratos 3-4 & $446(71.1)$ \\
\hline Estratos $5-6$ & $99(15.8)$ \\
\hline
\end{tabular}

dolor neuropático con base en la encuesta general, y después del examen clínico directo se diagnosticó finalmente a 16 de ellas (2.5\%) como probable dolor neuropático.

Cuando se evaluó el dolor crónico solo en mujeres (n: 422), se encontró DCNo en $36.9 \%$ (n: 156) y DNe en $3.3 \%$ (n: 14). Al comparar la frecuencia ponderada por género para ambos tipos de dolor, se halló una diferencia significativa a favor del femenino, con un $40 \%$ contra un $20 \%$ del masculino $(\mathrm{p}<0.001)$. En cuanto a la ocupación, las categorías denominadas "ama de casa, desempleado y pensionado", predominaron frente a las demás ocupaciones para el DCNo $(\mathrm{p}=0.006)($ Tabla 2).

Comparados los rangos etáreos de manera ponderada, se apreció una tendencia en ascenso del DCNo a medida que aumentó la edad $(\mathrm{p}<0.05)$ alcanzando una frecuencia de $50 \%$ en los mayores de 74 años (Tabla 3 ).

La localización del dolor crónico por regiones anatómicas mostró para el DCNo un franco predominio del dolor de cabeza con $36 \%(\mathrm{p}<0.05)$, seguido por MMIIS, región lumbar y MMSS, en orden descendente de frecuencia, mientras que

Tabla 2. Distribución porcentual de la población según características sociodemográficas de personas con dolor crónico nociceptivo (DCNo) y dolor crónico neuropático (DCNe).

\begin{tabular}{|c|c|c|}
\hline $\begin{array}{l}\text { Variables } \\
\text { sociodemográficas }\end{array}$ & $\begin{array}{l}\text { DCNo }(n=197) \\
\text { n }(\%)\end{array}$ & $\begin{array}{l}\text { DCNe }(n=16) \\
\text { n }(\%)\end{array}$ \\
\hline $\begin{array}{l}\text { Género } \\
\text { Femenino } \\
\text { Masculino }\end{array}$ & $\begin{array}{l}156(79.2) \\
41(20.8)\end{array}$ & $\begin{array}{l}14(87.5) \\
2(12.5)\end{array}$ \\
\hline $\begin{array}{c}\text { Edad / rango de edad } \\
\quad<25 \\
25-34 \\
35-49 \\
50-64 \\
65-74 \\
=>75\end{array}$ & $\begin{array}{l}17(8.6) \\
23(11.7) \\
58(29.4) \\
73(37.1) \\
12(6.1) \\
14(7.1)\end{array}$ & $\begin{array}{l}0(0.0) \\
0(0.0) \\
4(25.0) \\
7(43.8) \\
4(25.0) \\
1(6.3)\end{array}$ \\
\hline $\begin{array}{l}\text { Estado civil } \\
\qquad \text { Casado } \\
\text { Soltero } \\
\text { Divorciado } \\
\text { Unión libre } \\
\text { Viudo }\end{array}$ & $\begin{array}{l}98(49.7) \\
44(22.3) \\
21(10.7) \\
18(9.1) \\
16(8.1)\end{array}$ & $\begin{array}{l}9(56.3) \\
2(12.5) \\
2(12.5) \\
1(6.3) \\
2(12.5)\end{array}$ \\
\hline $\begin{array}{l}\text { Ocupación } \\
\text { Ama de casa } \\
\text { Empleado } \\
\text { Independiente } \\
\text { Pensionado } \\
\text { Estudiante } \\
\text { Desempleado }\end{array}$ & $\begin{array}{l}95(48.2) \\
43(21.8) \\
24(12.2) \\
15(7.6) \\
12(6.1) \\
8(4.1)\end{array}$ & $\begin{array}{l}13(81.3) \\
0(0.0) \\
0(0.0) \\
2(12.5) \\
0(0.0) \\
1(6.3)\end{array}$ \\
\hline $\begin{array}{l}\text { Nivel educativo } \\
\text { Bachiller } \\
\text { Primaria } \\
\text { Profesional/Tecnólogo } \\
\text { Analfabeta }\end{array}$ & $\begin{array}{l}98(49.7) \\
57(28.9) \\
41(12.2) \\
1(0.5)\end{array}$ & $\begin{array}{l}10(62.5) \\
5(31.3) \\
1(6.3) \\
0(0.0)\end{array}$ \\
\hline $\begin{array}{l}\text { Estrato } \\
\qquad \begin{array}{l}\text { Estratos } 1 \text { - } 2 \\
\text { Estratos } 3 \text { - } 4 \\
\text { Estratos } 5 \text { - } 6\end{array}\end{array}$ & $\begin{array}{l}36(18.3) \\
125(63.5) \\
36(18.3)\end{array}$ & $\begin{array}{l}1(6.3) \\
13(81.3) \\
2(12.5)\end{array}$ \\
\hline
\end{tabular}


en el DCNe prevaleció el dolor en MMSS con un 75\%, con menor representatividad de las otras localizaciones (Tabla 4).

La duración del DCNo fue mayor a un año en el $66.5 \%$ (n: 131) y superior a cinco años en el 35\% (n: 69) de los casos. Para el DCNe la duración fue mayor a un año en el $93.7 \%$ (n: 15) y superior a cinco años en el $25 \%$ (n: 4) de los casos. No hubo diferencias estadísticas significativas de duración para cada tipo de dolor ( $\mathrm{p}>0.05)$.

En ambos tipos de dolor crónico la intensidad fue calificada como "moderada a severa" de manera similar en cerca del $75 \%$ de los casos, en tanto que el descrito como “intolerable" predominó claramente en el DCNe (Figura 1).

En lo que respecta a la repercusión del dolor crónico sobre el estado de ánimo, el $16.7 \%$ de pacientes con DCNo y el $31.2 \%$ con DCNe, manifestaron no haberse afectado, mientras que los afectados en grado referido como "moderado" o "severo" fue reportado por la mitad de los encuestados, para ambos tipos de dolor. Hubo diferencia estadística significativa cuando se compararon afectados y no afectados en su ánimo para ambos tipos de dolor $(\mathrm{p}<0.05)$.

En cuanto a la repercusión sobre la ansiedad, el $27.9 \%$ de los pacientes con DCNo y el $18.7 \%$ de los pacientes con DCNe expresaron no haberse comprometido, mientras que una repercusión "moderada" o "severa", se expresó en cerca de la mitad de los sujetos para ambos tipos de dolor. Cuando se compararon afectados y no afectados por ansiedad para ambos tipos de dolor, se halló también una diferencia con significancia estadística $(\mathrm{p}<0.05)$ (Tabla 5).

En lo atinente al efecto del dolor crónico sobre el sueño, éste se vio afectado en $36.5 \%(n=72)$ de los encuestados con DCNo y en $43.8 \%(n=7)$ de los que presentaron DCNe.

$\mathrm{El} 41 \%$ de los encuestados reportó la práctica de autoformulación habitual para su dolor crónico, sin diferencia entre los dos tipos de dolor.

Tabla 3. Frecuencia de tipos de dolor por rangos de edad.

\begin{tabular}{|l|c|c|}
\hline Rangos etáreos & $\begin{array}{c}\text { DCNo } \\
\text { Encuestados (n/N) }\end{array}$ & $\begin{array}{c}\text { DCNo } \\
\text { Valor ponderado (\%) }\end{array}$ \\
\hline$<25$ a & $17 / 93$ & 18.2 \\
$25-34$ a. & $23 / 89$ & 25.8 \\
$35-49$ a. & $58 / 178$ & 32.5 \\
$50-64$ a & $73 / 190$ & 38.4 \\
$65-74$ a. & $12 / 49$ & 24.4 \\
75 a y $>$ & $14 / 28$ & 50 \\
\hline
\end{tabular}

Tabla 5. Repercusión porcentual del dolor en el estado de ánimo y en la ansiedad.

\begin{tabular}{|l|lcl|lc|}
\hline \multirow{2}{*}{ Intensidad } & \multicolumn{2}{|c|}{ Ánimo n (\%) } & & \multicolumn{2}{c|}{ Ansiedad n (\%) } \\
\cline { 2 - 3 } & DCNo & DCNe & & DCNo & DCNe \\
\hline MEVE & $64(32.5)$ & $3(18.7)$ & & $47(23.8)$ & $5(31.2)$ \\
SEVERA & $60(30.5)$ & $3(18.7)$ & $53(26.9)$ & $3(18.7)$ \\
& $40(20.3)$ & $5(31.2)$ & $42(21.3)$ & $5(31.2)$ \\
\hline
\end{tabular}

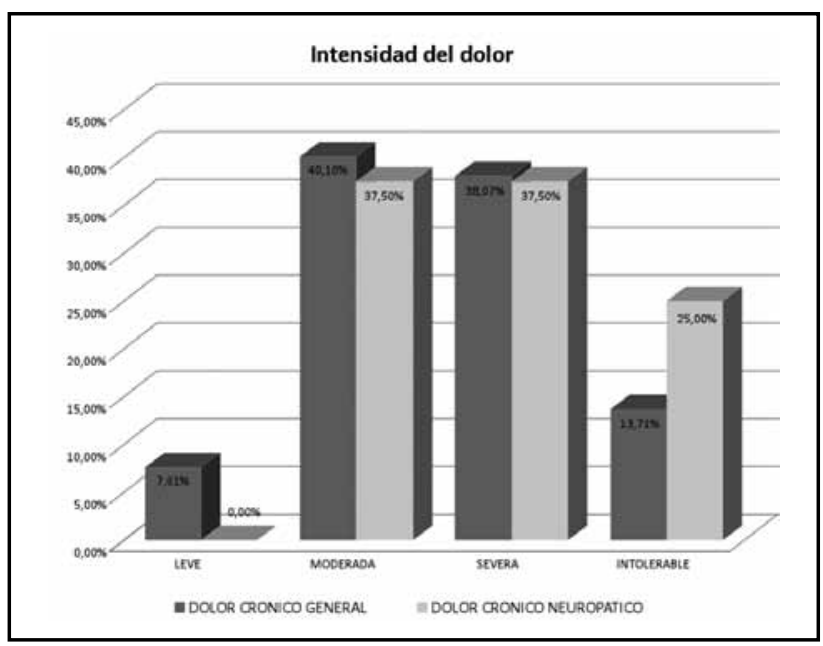

Figura 1. Intensidad del dolor en DCNo y DCNe.

En cuanto al uso de analgésicos para ambos tipos de dolor crónico se destacó el predominio del consumo de acetaminofen y de AINES sin grandes diferencias en su frecuencia de uso, y la escasa utilización de opioides para ambos tipos de dolor (Tabla 6).

Cuando se aplicó a los sujetos con dolor crónico la escala de bienestar OMS-5 con punto de corte de 13, se detectó que el $19.7 \%(n=42)$ de ellos obtuvo un puntaje compatible con un bajo nivel de bienestar emocional.

Los resultados obtenidos con la aplicación de la escala de dolor neuropático DN4, se discriminaron para puntajes totales sobre 10 , y sobre 7 con puntos de corte de cuatro o más y de tres o más, respectivamente. Con la aplicación de la escala DN4 sobre 10 , se identificaron $81.2 \%$ de los ca-

Tabla 4. Frecuencia de la localización del dolor crónico.

\begin{tabular}{|l|c|c|}
\hline Localización & $\begin{array}{c}\text { Dolor crónico } \\
\text { nociceptivo } \\
\text { DCNo }(\%)\end{array}$ & $\begin{array}{c}\text { Dolor crónico } \\
\text { neuropático } \\
\text { DCNe }(\%)\end{array}$ \\
\hline Cabeza & $71(36.0)$ & $1(6.3)$ \\
Miembros inferiores & $40(20.3)$ & $3(18.8)$ \\
Lumbar & $30(15.2)$ & $4(25.0)$ \\
Miembros superiores & $24(12.2)$ & $12(75.0)$ \\
Abdomen & $20(10.2)$ & $0(0.0)$ \\
Hombros & $6(3.0)$ & $1(6.3)$ \\
\hline
\end{tabular}

Tabla 6. Uso de analgésicos en DCNo y DCNe.

\begin{tabular}{|l|c|c|}
\hline Analgésicos & (DCNo) n (\%) & (DCNe) n (\%) \\
\hline Acetaminofén & $110(55.8)$ & $8(50.0)$ \\
AINES & $105(53.3)$ & $9(56.3)$ \\
Opioides débiles & $4(2.0)$ & $2(12.5)$ \\
Otros & $27(13.7)$ & $1(6.3)$ \\
\hline
\end{tabular}


sos con DCNe, mientras que cuando se aplicó dicha escala sobre 7, se identificaron $87.5 \%$ de ellos. Sin embargo, para el DCNo estas escalas identificaron erróneamente $21.3 \%$ (DN4/10) y $34.5 \%(\mathrm{DN} 4 / 7)$ de personas como sugestivas de dolor neuropático.

Evaluada la validez de las pruebas se observó para la escala DN4 sobre 10, una sensibilidad del 81.2\% (IC 95\% 53.7-95.0) y una especificidad de 78.7\% (IC 95\% 72.2-84,0), con un valor predictivo positivo (VPP) de $23.6 \%$ (IC $95 \%$ 13.7-37.3) y valor predictivo negativo (VPN) de $98.1 \%$ (IC 95\% 94.1-99.5). Al analizar la escala DN4 sobre 7, se obtuvieron los siguientes resultados: sensibilidad: $87.5 \%$ (IC 95\% 60.4-97.8), especificidad: 65.5\% (IC 95\% 58.372.0), VPP: $17.1 \%$ (IC $95 \%$ 10.0-27.3) y VPN de $98.5 \%$ (IC 95\% 94.0-99.7).

Los pacientes identificados como probables casos de dolor crónico neuropático una vez examinados por el grupo investigador, mostraron los siguientes diagnósticos clínicos: mononeuropatía por atrapamiento del nervio mediano (síndrome del túnel carpiano) con $62.5 \%$, seguido de neuralgia postherpética con $12.5 \%$, neuropatía diabética con $12.5 \%$, radiculopatía con $6.3 \%$ y mononeuropatía del nervio radial con $6.3 \%$ (Tabla 7).

\section{Discusión}

El presente estudio permitió establecer la prevalencia y las características demográficas y clínicas del dolor crónico en una muestra representativa del área urbana de la ciudad de Manizales. La prevalencia encontrada fue de $33.9 \%$, cifra similar a la detectada en el año 2008 en el departamento de Caldas de 31\% (7). Las prevalencias publicadas en Europa y Norteamérica han oscilado entre $11.5 \%$ y $55.2 \%(2,3,18$, 19), mientras que en Latinoamérica los estudios poblacionales han mostrado en Cuba $40.3 \%$, en Chile $34.3 \%$ y en México $16.8 \%$ (20). Esta variabilidad en las cifras podría explicarse por las distintas metodologías empleadas para recoger la información, por diferencias en la definición de dolor crónico y de carácter étnico y geográfico, entre otras razones. Una revisión sistemática reciente (21) basada en la definición de dolor crónico de la IASP, mostró una prevalencia promedio de $35.5 \%$, similar a la encontrada en el presente estudio, lo cual indica que una tercera parte de la población adulta sufre esta condición potencialmente discapacitante.

En los tipos de dolor crónico se destacó el predominio del

Tabla 7. Distribución de diagnósticos clínicos en DCNe.

\begin{tabular}{|l|c|}
\hline Diagnóstico clínico & Dolor crónico neuropático n (\%) \\
\hline Neurop. Stcarpo & $10(62.5)$ \\
Neuralg-Posther & $2(12.5)$ \\
Neurop. Diabética & $2(12.5)$ \\
Neurop. Radial & $1(6.3)$ \\
Neurop-Radiculop L5 & $1(6.3)$ \\
\hline
\end{tabular}

dolor nociceptivo sobre el dolor neuropático y para ambos tipos hubo un predominio estadísticamente significativo del género femenino (mujeres $40 \%$ y hombres $20 \%$ ), lo cual muestra una tendencia parecida con lo reportado en la revisión de la IASP antes mencionada, donde el dolor crónico afectó al 39.6\% de las mujeres y al 31\% de los hombres. Así mismo, el presente estudio mostró una franca tendencia a mayor prevalencia de dolor crónico con el aumento en años, siendo más evidente en las personas de 75 y más años con un $50 \%$, coincidente con lo informado por Helme R.D. y cols. (22). Esta situación posiblemente se explica por la mayor frecuencia de enfermedades degenerativas y comorbilidades en la tercera edad.

En lo que respecta a localizaciones anatómicas del dolor crónico, vale la pena destacar el predominio de la cefalea en alrededor de la tercera parte de nuestros encuestados, lo cual coincide con lo publicado a nivel nacional por la ACED $(5,6)$. Como es bien sabido, existen múltiples etiologías del dolor de cabeza siendo las dos más importantes la cefalea tipo tensión y la migraña, las cuales tienen frecuente tendencia a la cronificación. Además, en los últimos años ha adquirido destacada importancia la cefalea crónica diaria asociada a sobreuso de analgésicos, condición esta que ha sido poco estudiada en nuestro medio. Estudios nacionales neuroepidemiológicos realizados en Jamundí (Valle) en 1984 (23) y en Piedecuesta (Santander) en 1995 y 2002 $(24,25)$ encontraron prevalencias para migraña de $23.7 \%$, $20.5 \%$ y $19.4 \%$, respectivamente. Un estudio sobre prevalencia de enfermedades neurológicas en el departamento de Caldas en el año 2004 (26) detectó cefalea tensional en $33.5 \%$, migraña en $27.8 \%$ y cefalea crónica diaria en $9.7 \%$ de los sujetos encuestados. Una investigación realizada en Bucaramanga (Santander) en el año 2008, reportó una prevalencia de cefalea crónica diaria de $8.4 \%$ (27). Los estudios anteriores nos permiten apreciar la notable frecuencia que posee el dolor de cabeza en nuestro medio y la necesidad de seguir identificando factores de riesgo y comportamiento epidemiológico de esta patología.

Los datos obtenidos en cuanto a duración en años del dolor crónico mostraron que algo más de la tercera parte de los sujetos lo había tenido por más de cinco años. En un estudio epidemiológico noruego (28) mediante encuesta postal, se documentó una duración similar en $65 \%$ de los sujetos. La intensidad del dolor calificada como moderada o severa por las dos terceras partes de los individuos se puede considerar alta, lo que sumado a la cronicidad antes anotada, se constituye en una situación de riesgo de discapacidad y deterioro de la calidad de vida.

Aunque no se aplicaron escalas específicas de tamizaje para depresión o ansiedad, la encuesta reveló que la mitad de los sujetos tenía una repercusión entre moderada y severa del dolor para ambos estados emocionales. Cuando se evaluó la percepción de bienestar emocional (OMS-5), uno de cada cinco sujetos mostró una puntuación compatible con trastorno depresivo, lo cual refuerza la necesidad de tener en 
cuenta los aspectos emocionales en esta condición. Como se sabe, la depresión es común en pacientes con dolor crónico, con prevalencias que varían ampliamente desde $1.5 \%$ hasta 87\% (29), dependiendo de la población estudiada y de los instrumentos y criterios empleados para su diagnóstico. Con relación a la ansiedad, un estudio encontró que $35 \%$ de la población con dolor crónico cumplía criterios para trastorno de ansiedad, comparado con 18\% de los controles (30).

Otro aspecto de la encuesta que se informó alterado fue el patrón de sueño de tipo insomnio, en poco más de la tercera parte de los sujetos. Algunos estudios han reportado que la prevalencia de alteraciones del sueño en personas con dolor crónico puede oscilar entre 50-70\% $(31,32)$. Aunque en el presente estudio el porcentaje de insomnio fue menor al de los estudios anteriores, sigue siendo significativo y es un aspecto que requiere atención en el abordaje del paciente con dolor crónico.

En los pacientes con dolor crónico es relativamente frecuente la autoformulación, como se ha encontrado en dos importantes estudios europeos con valores que oscilan entre 29 y $50 \%(3,19)$. En el presente estudio la autoprescripción (41\%) se ubicó dentro del rango anterior y fue igual al resultado obtenido en el estudio de dolor crónico en el departamento de Caldas (2008) (7). Este comportamiento es preocupante por las implicaciones que tiene para el manejo de una condición crónica que tiende a mantenerse en el tiempo y que expone a las personas a efectos adversos de los fármacos, que pueden llegar a ser graves. Aunque son numerosos los factores que inducen a la autoformulación, algunos tan obvios como los socioeconómicos y culturales, resulta necesario adelantar estudios específicos sobre esta práctica habitual.

Otro resultado llamativo del presente estudio fue el manejo del dolor crónico con antiinflamatorios no esteroideos (AINES) en más de la mitad de los casos, en contraposición a un número muy bajo en el uso de opioides y de medicamentos coadyuvantes. Sin embargo, ésto no parece ser una particularidad de nuestro medio, pues en el estudio de Breivik en Europa también se menciona el uso de AINES en alrededor del $50 \%$ de los casos, si bien el uso de opioides fue un poco mayor que en el presente estudio (3). Es de resaltar el riesgo elevado del uso prolongado de AINES debido a las potenciales reacciones adversas a nivel gastrointestinal, cardiovascular y renal, que los hace poco indicados para manejo del dolor crónico, situación que puede empeorar si son autoprescritos.

Una de las herramientas más populares para tamizaje de dolor neuropático, tanto a nivel individual como en estudios poblacionales, es la denominada Escala de Dolor Neuropático DN4, la cual ha sido traducida a diferentes idiomas y exhibe adecuada sensibilidad y especificidad (33).

En este estudio se empleó dicha escala para identificar a los sujetos sospechosos de dolor neuropático, los cuales fueron evaluados posteriormente para confirmar o desechar este tipo de dolor crónico. Cuando analizamos el comporta- miento de esta escala en su versión original sobre 10 puntos y en la versión modificada sobre 7 puntos, encontramos que en esta última la sensibilidad de la prueba no difiere significativamente de la versión sobre 10 puntos $(87.5 \%$ vs $81.2 \%$ ) mientras que la especificidad sí se afecta un poco más $(65.5 \%$ vs $78.7 \%)$. Esta situación parece importante puesto que puede explicar las diferencias encontradas en la prevalencia de dolor neuropático en población general de acuerdo con el método de recolección de información y la manera de aplicación de la escala, bien si ésta se hace mediante examen directo del sujeto o si se realiza mediante encuesta por correo postal, correo electrónico o entrevista telefónica, en las cuales no hay oportunidad de corroborar con el examen físico los síntomas subjetivos de la persona. El estudio de Bouhassira y cols. (2008) (14), denominado STOPNEP (Study of the Prevalence of Neuropathic Pain) uno de los más grandes realizados hasta la fecha con 23.712 personas encuestadas, evaluó la prevalencia de dolor crónico con y sin características neuropáticas en población francesa mediante encuesta postal. La prevalencia de dolor crónico fue de $31.7 \%$, similar a la prevalencia del presente estudio de $33.9 \%$, en tanto que la prevalencia de dolor con características neuropáticas fue de $6.9 \%$, más alta que la nuestra de $2.5 \%$, lo que podría explicarse por la menor especificidad que exhibe la escala DN4 sobre siete. Observaciones similares pueden hacerse para el estudio de Torrance (2006) en el Reino Unido, efectuado con metodología de encuesta postal utilizando la escala para tamizaje de dolor neuropático denominada S-LANSS ( Leeds Assessment of Neuropathic Symptom and Signs Score), el cual encontró una prevalencia de dolor neuropático de $8.2 \%$ (2).

Como reconoce Bouhassira en su artículo, es posible que una proporción de sus encuestados haya tenido dolor crónico con y sin características neuropáticas, es decir un "dolor mixto", lo cual nos lleva a concluir que la prevalencia real de dolor neuropático pudiera ser menor a la informada en su estudio. Estos dos estudios nos permiten aseverar que la prevalencia real del DCNe deba ser estimada solo cuando se da una evaluación clínica que permita confrontar los síntomas sugestivos contra unos signos más específicos de este tipo de dolor.

Los trabajos previos de Bowsher (34), Dworkin (35) e Irving (36) reportan prevalencias de dolor neuropático de 1 a $3 \%$ y, en la recién publicada guía para el diagnóstico y manejo del dolor neuropático elaborada por un grupo de expertos latinoamericanos (2008) (16), se cita una prevalencia de dolor neuropático en Latinoamérica de $2 \%$, basada en el trabajo de Guevara (15), siendo todas estas cifras similares a la prevalencia hallada en el presente estudio.

Con relación a los diagnósticos clínicos de dolor crónico neuropático, no se identificó ninguno de origen central y todos correspondieron a patologías de daño nervioso periférico, con franco predominio del causado por atrapamiento del nervio mediano, lo cual correspondió a $1.6 \%$ de la muestra. En un estudio de prevalencia de síndrome de túnel 
carpiano en población general, se encontró que 3.8\% tenían un diagnóstico clínico y $2.7 \%$ tenían diagnóstico clínico y electrofisiológico de éste (37). De todas maneras, se debe reconocer que el número de sujetos con dolor neuropático resultó escaso para identificar una distribución etiológica más representativa de este tipo de dolor. Debemos tener en cuenta que para determinar la causa de una lesión neurológica se requiere de un examen físico completo y con cierta frecuencia de estudios electrofisiológicos, pruebas de laboratorio o exámenes radiológicos, los cuales no son fáciles de implementar en estudios epidemiológicos.

En conclusión, el presente trabajo es un aporte al conocimiento en nuestro país sobre la epidemiología del dolor crónico y algunos aspectos del dolor de tipo neuropático. Fuera de los datos aportados por la ACED, no se encuentran publicaciones nacionales que aborden este tipo de información y, en consecuencia, consideramos de la mayor importancia que se sigan realizando trabajos que arrojen datos más precisos sobre la etiología del dolor crónico nociceptivo y neuropático e identifiquen factores de riesgo, comorbilidades e impacto sobre calidad de vida en la población general.

\section{Financiación}

Vicerrectoría de Investigaciones y Posgrados de la Universidad de Caldas y convenio de cooperación entre Universidad de Caldas y Dirección Territorial de Salud de Caldas.

\section{Conflictos de interés}

Ninguno para declarar.

\section{Agradecimientos}

- A la Dirección Territorial de Salud de Caldas por la cofinanciación de la investigación.

- Al doctor Arnoby Chacón Cardona, jefe del Departamento Clínico por la revisión del documento y aportes en el análisis estadístico de los resultados.

- A la doctora María Victoria Benjumea, directora del Instituto de Investigaciones de la Facultad de Ciencias para la Salud de la Universidad de Caldas, por su valioso aporte en el análisis de datos.

\section{Referencias}

1. Subcommittee of Taxonomy of the International Association for the Study of Pain. www.iasp pain.org/AM/Template.

2. Torrance N., Smith BH, Bennett MI, and Lee AJ. The epidemiology of chronic pain of predominantly neuropathic origin. Results from a general population survey. J Pain, 7: 2006: 281-9.

3. Breivik H., Collett B., Ventafridda V., Cohen R. and Gallacher D. Survey of chronic pain in Europe: Prevalence, impact on daily life, and treatment. Eur $J$ Pain 2006; 10: 287-333.

4. Bassols A, Bosch F, Campillo M, Canellas M, Banos JE. An epidemiological comparison of pain complaints in the general population of Catalonia (Spain. Pain 1999; 83: 9-16.

5. III Encuesta Nacional del Dolor. Asociación Colombiana para el estudio del dolor(ACED.. Abril de 2004. www.urosario.edu.co/investigación/ tomo1/ fasciculo8.

6. IV Encuesta Nacional del Dolor. Asociación Colombiana para el estudio de dolor (ACED. Abril 2008. Ponencia Dr. John Jairo Hernández. XVII Congreso Internacional de Dolor. Bogotá (Col). 2008.

7. Díaz R, Marulanda F, Saenz X. Estudio epidemiológico del dolor crónico en Caldas, Colombia (Estudio DOLCA. Acta Med Colomb 2009; 34: 96-102.

8. Merskey H, Bogduk N. Classification of chronic pain. Seattle: IASP Press, 1994.

9. Treede RD, Jensen TS, Campbell JN, Cruccu G, Dostrovsky JO, Griffin JW, et al. Neuropathic pain. Redefinition and a grading system for clinical and research purposes. Neurology 2008; 70: 1630- 5.
10. Bennett GJ. Neuropathic pain: new insights, new interventions. Hosp Pract 1998 33: $95-104$

11. Ruiz F, Carrasco ML. El dolor neuropático en la consulta médica en América Latina: resultado de una encuesta en cinco países. En: Guías para el diagnóstico y manejo del dolor neuropático: consenso de un grupo de expertos latinoamericanos. Rev Iberoamericana de Dolor 2008; 2: 15-48.

12. Bowsher D. Neurogenic pain syndromes and their management. Br Med Bull 1991; 47: 644-66.

13. Gilron I, Watson PN, Cahill CM, Moulin DE. Neuropathic pain: a practical guide for the clinician. CMAJ 2006; 175: 265-75.

14. Bouhassira D, Lantéri-Minet, Attal N, Laurent, B,Touboul Ch. Prevalence of chronic pain with neuropathic characteristics in the general population. Pain 2008; 136: 380-7.

15. Guevara U, Covarrubias A, García G, Hernández S. Grupo de Consenso para el Manejo del Dolor Neuropático. [Practice guidelines for neuropathic pain management]. Rev Invest Clin. 2006; 58: 126-38.

16. Acevedo JC, Amaya A, Casasola OL, Chinchilla N, De Giorgis M, Flórez S et al. Guías para el diagnóstico y el manejo del dolor neuropático: consenso de un grupo de expertos latinoamericanos. Rev Iberoamericana del Dolor 2008; 2: 15-46.

17. Nicholson B., Verm S. Comorbidities in Chronic neuropathic.pain. Pain Medicine 2004; 5: 9 .

18. Català E, Reig E, Artés M, Aliaga L, López JS, Segú JL. Prevalence of pain in the Spanish population: telephone survey in 5000 homes. Euro J Pain 2001; 5: $1-8$.

19. Bassols A, Bosch F, Baños JE. How does general population treat their pain? A survey in Catalonia (Spain.. J Pain Symptom Manage 2002; 23: 318-28.

20. Bistre S. El Dolor Crónico en América Latina Rev. Iberoamericana del Dolor 2007; 3: 3-5.

21. Carr DB. How prevalent is chronic pain? Pain Clinical Updates. International Association for the Study of Pain 2003. Vol XI, $\mathrm{N}^{\circ} 2$.

22. Helme RD, Gibson SJ. In: Jensen TS, et al. (Eds.. Proceedings of the 8th World Congress on Pain, Progress in Pain Research and Management, Vol. 8. Seattle: IASP Press, 1997, p919-44.

23. Zúñiga A, Antolínez BR. Prevalencia de enfermedades neurológicas en Jamundí, Valle, Colombia, 1984. Colombia Médica 1986; 17: 151-3.

24. Pradilla G, Vesga BE, Bautista LE. Neuroepidemiología en Piedecuesta, población semiurbana de Santander. Acta Med Colomb 2000; 25: 286.

25.Pradilla G, Vesga B. Estudio neuroepidemiológico en Piedecuesta (Santander). Acta Med Colomb 2002; 27: 407-20.

26. Díaz-Cabezas R, Ruano-Restrepo MI, Chacón-Cardona JA, Vera-González A. Perfil neuroepidemiológico en la zona centro del departamento de Caldas (Colombia), años 2004-2005. Rev Neurol 2006; 43: 646-52.

27. Rueda-Sánchez M., Díaz-Martínez L.A. Prevalence and associated factors for episodic and chronic daily headache in the Colombian population. Cephalalgia 2008; 28: 216-25.

28. Rustøen T, Wahl AK, Hanestad BR, Lerdal A, Paul S, Miaskowski C. Prevalence and characteristics of chronic pain in the general Norwegian population. Eur J Pain 2004; 8: 555-65.

29. Carr D. Pain in depression- depression in pain. Pain Clinical Update (IASP) 2003; Vol XI, No 5 .

30. McWilliams L, Cox BJ, Enns MW. Mood and anxiety disorders associated with chronic pain: an examination in a nationally representative sample. Pain 2003, 106: $127-133$.

31.Pilowsky I, Crettenden I, Townley M. Sleep disturbance in pain clinic patients Pain 1985;23:27-33.

32. Morin CM, Gibson D, Wade J. Self-reported sleep and mood disturbance in chronic pain patients. J Clin Pain 1998; 14: 311-4.

33. Bouhassira D, Attal N, Alchaar H, Boureau F, Brochet B, Bruxelle J, et al. Comparison of pain syndromes associated with nervous orsomatic lesions and development of a new neuropathic pain diagnostic questionnaire (DN4). Pain 2005; 114: 29-36.

34. Bowsher D. Neurogenic pain syndromes and their management. Br Med Bull 1991; 47: 644-66.

35. Dworkin RH, Backonja M, Rowbotham MC, Allen RR, Argoff CR, Bennett GJ, et al. Advances in neuropathic pain: diagnosis, mechanisms, and treatment recommendations. Arch Neurol 2003; 60: 1524-34.

36. Irving GA. Contemporary assessment and management of neuropathic pain Neurology 2005; 64: 21-27.

37. Atroshi I, Gummesson C, Johnsson R, Ornstein E, Ranstam J, Rosén I Prevalence of Carpal Tunnel Syndrome in a General Population. JAMA 1999; 282: $153-8$. 